\title{
Europe: History, Current Situation and Control Measures for Infectious Bronchitis
}

Workshop: Infectious Bronchitis (IB) in the Brazilian Poultry Industry

\section{Author(s)}

Jones RC

School of Veterinary Science

University of Liverpool

Leahurst Campus, South Wirral, UK

\section{ABSTRACT}

The emergence and nature of different strains of infectious bronchitis virus (IBV) in Europe are described. Infectious bronchitis (IB) is the most important endemic viral respiratory disease where highly pathogenic Newcastle disease and avian influenza are not present. IB was first described in the UK in 1948 and identified as Massachusetts type. In the 1970s and 80s new serotypes were reported in Holland and elsewhere and new vaccines were developed. The 1990s saw the emergence of the major variant commonly called 793B, again needing a new vaccine. Two novel types have been recognised since 2000 , Italy 02 and QX. Italy 02 appears to be well controlled by the use of two different live vaccines (H120 and the 793B-related 4/91) while for QX, associated with nephritis in young birds and silent layers, new vaccines are in development. The use of two vaccines as above is a widely used protocol and is capable of protecting against a wide range of different types. Alternative approaches to IB vaccination are discussed. The importance of constant surveillance for prevalent and novel IBV types is emphasised and the value of experimental infections in chickens to determine the pathogenesis and pathology of new types in addition to testing efficacy of vaccines is outlined.

\section{INTRODUCTION}

The European Union (EU) currently comprises 27 states and because barriers are so relaxed, disease affecting one country is likely to spread to another. Thus, an appraisal of infectious bronchitis (IB) in Europe can consider the EU as a single state, although there are variations in the different nations. This presentation describes the history of IB in Europe and the appearance of new types of virus which have influenced control measures of this disease. The main problem with IB is the variability of the virus, a Type 3 coronavirus. The frequent emergence of variants associated with changes in the S1 spike gene occur due to mutations, recombinations and sometimes introduction of virus from other regions.

\section{HISTORY OF IBV TYPES IN EUROPE}

\section{8-1980s}

IB was first described in the USA in the 1930s and was identified in the UK in 1948 (Asplin, 1948). For many years it was assumed that the Massachusetts serotype was the only one present in Europe, presumably this virus having been transferred from the USA in infected birds. However, in the 1980s, it was demonstrated in the Netherlands that disease outbreaks could occur in flocks vaccinated against IB with $\mathrm{H} 120$ or H52 commercial products (Davelaar et al., 1984). These were the only vaccines available at the time and were derived from a Mass-type 
virus isolated from Huyben's farm and passaged 100 or 52 times in fertile eggs (Bijlenga et al., 2004). By cross neutralisation tests in eggs, these viruses were shown to be different to the common American types. Four serotypes were described, D207, D212, D3128 and D3896. H120 was not protective against these types in experimental infections. Three of these serotypes were present in the UK and presumably elsewhere in Europe. The origin of these variants is unknown but vaccine pressure may have been influential.

Soon after this, Kusters et al. (1987), using T1 fingerprinting of viral RNA, demonstrated two main clusters of these new IBVs. Cluster 1 contained vaccines $\mathrm{H} 52$ and $\mathrm{H} 120$ and field viruses D387, V1385, V1397. Cluster 2 contained D274, D212, D1466, D3128, and D3896, of which D274 and D1466 were developed as vaccines. This report was one of the first using molecular methods to differentiate between IBVs, which hitherto had been classified by crossneutralisations in eggs. D2704 and D1466 were both detected in several countries in the Western European IBV survey undertaken between 2002 and 2006 (Worthington et al., 2008).

\section{0s}

Early in this decade, reports described a new type of IBV in broiler, layer and breeder chicken flocks in the UK (Parsons et al., 1992) This type, known most commonly as 793B but also 4/91 or Cr88 was shown to have a nucleotide sequence in the hypervariable regions of the $\mathrm{S} 1$ spike gene quite distinct from Massachusetts and Dutch variant viruses. H120 was not effective against this variant. Initially, field infection with this genotype was associated with enteritis, slower spread than usual with IB and pectoral myopathy. An experimental study (Dhinakar Raj and Jones 1997) however, showed the virus to behave like other IBVs except that virus replicated in the gut for longer than in the respiratory tract. The origin of this virus is uncertain but it shares an S1 gene spike sequence of 96\% with strain G isolated in Morocco in 1986 (C. J. Naylor and R. C. Jones, unpublished). 793B types have been the predominant genotype in Europe in the earlier years of this decade.

This virus has been shown to be very widespread in global distribution (Cook et al., 1996), virtually everywhere except USA and Australia. Live vaccines have been developed (4/91 and IBV88) and are used as a routine in Europe in vaccine programmes. Indeed a landmark paper (Cook et al., 1999) showed that the combination of $\mathrm{H} 120$ at day old and 4/91 at 14 days provides wide protection against a range of different IBVs. This combination has been adopted widely in the face of several important variant viruses. This is referred to again later.

\section{0 to the present}

In the present decade, two important variants have emerged in Europe. The first, called Italy 02 and described in Italy, is likely to have been in Europe several years before the '02' label (Dolz et al., 2006). Its origins are unknown but it has been widespread in Europe (Worthington et al., 2008). It is genetically distinct from Mass and other types and does Masstype vaccines are not effective against it. It causes disease consistent with relatively mild IB.

Italy 02 appeared to reach a peak of prevalence in Western Europe in 2003 but since then has been in decline (Worthington et al., 2008) although it replaced $793 B$ as the predominant genotype in Spain (Dolz et al., 2006). It has been the most prevalent wild-type in Europe.

The second new genotype to emerge has been called QX (D388 in Holland). This is a virus with near $100 \%$ S1 spike sequence identity with QX virus first reported in China in 1996 and causing proventriculitis. In Europe it was detected around 2002 and has been a serious cause of disease, namely nephritis in young birds, resulting in significant mortalities and 'silent layers' in mature females. The silent layer syndrome recalls work done in the 1970s where infection of baby chicks with certain viruses (including Mass types) with no maternal antibodies, can cause damage to the oviduct such that at sexual maturity, the oviduct does not remain patent and large cysts develop (see Dhinakar Raj and Jones, 1997). Ovum production continues normally but no external eggs are laid. Such affected birds can seriously compromise the overall production of the flock.

An interesting aspect of this virus is that although it has travelled from China across Asia and Europe in a time span similar to that which brought highly pathogenic avian influenza (HPAl) via the same route, there is no known wild avian species known to transport IBVs over large distances. Despite this, having reached Europe, QX has spread to the extremities of the continent slowly, being detected in Spain and UK only in 2008.

Experimentally and in other trials, the dual vaccination protocol (De Wit and Van der Sande 2009; Jones R. C., unpublished) is effective in countering QX 
infection but because of the severity of disease and its manifestations, calls for a dedicated vaccine have been heeded by the vaccine industry and these are in development.

Thus two major variants have emerged in Europe since 2000, but while one is relatively mild in effects, the other, apparently spreading more slowly within Europe, is much more severe in disease manifestations. QX clearly has particular tropisms for the kidneys and oviduct epithelium.

\section{Current methods of Control in Europe}

In the EU, current control is centred on the use of Mass and major variant (793B)-type live and killed vaccines. Mass-type vaccines used are H120, MM (modified Mass) and Ma5. The 794B-type vaccines are 4/91 and CR88. These are given to birds during the growing period as monovalent vaccines, with timing frequently involving Mass followed by 793B type and resulting in wide protection.

One manufacturer produces bivalent live vaccines: IB primer, comprising $\mathrm{H} 120$ and Dutch variant D274 and IBMM+Ark. The second of these is unusual in that a non-indigenous vaccine (American ARK) has been licensed in some countries since it offers protection against 793B types (Jones and Worthington, unpublished). However, some countries, notably France, do not allow bivalent vaccines, fearing that simultaneous administration could result in potentially dangerous recombination. No evidence of this has yet been reported.

As mentioned above, the double vaccine protocol is very widely used and is likely to have influences the decline of Italy 02 in Europe. However, it is likely that $Q X$ vaccines will soon be available.

Inactivated IB vaccines are given prior to point of lay often in combination with other inactivated viruses. Live vaccines are occasionally given to flocks in lay.

\section{Observation on approaches to IBV vaccination- how to deal with variants?}

In regions where licensed vaccines are from the same genotype as the field challenge, then IB vaccines work well. However, where variants appear and persist and against which existing vaccines do not protect, then this presents a big problem. A number of possibilities exist for addressing the problem of a new important variant for which a new vaccine is perceived to be necessary.

1. Test the existing repertoire of vaccines. Sometimes protection can be offered by an unrelated vaccine. For example, D274 vaccine protects against the unrelated 793B variant (Dhinakar Raj and Jones, 1996).

2. Develop an empirical vaccine by attenuation in eggs or perhaps cell culture. This is the traditional 'fire brigade' method but is a long tedious process.

3. Use molecular techniques to engineer a vaccine for the new challenge virus that will not revert to virulence. Recent examples of this are modified DNA vaccination and a multiepitope-based peptide vaccine. One of the most promising of the new generation of vaccine appears to be the 'spike swapping' technology, whereby using reverse genetics, an infectious clone is produced into which specific S1 spike genes can be incorporated, appropriate to the new variant (Casais et al., 2001). Variations on this theme have also included incorporation of nucleocapsid genes or specific cytokines to induce a broader immunity (Cavanagh et al., 2007).

4. Use two heterologous IBV vaccines (Cook et al., 1999). This method has been shown to be very successful, even though the mechanisms have not been elucidated to date. It is usual to first administer a Massachusetts-type vaccine followed by a variant (793B-type in Europe) offers wide protection against types which are different again. Such a programme has been shown to be efficacious against for example Italy 02 and QX (Jones et al., 2005; De Wit and van de Sande, 2009).

5. In ovo vaccination. This is under development (Tarpey et al., 2006) and is dependent on the strain of IBV not killing embryos.

\section{Importance of surveillance}

Central to control of IB and the big problem of variants is constant surveillance. Formerly, identification of types requires the tedious procedures of virus isolation followed by cross neutralisation with specific antisera. Now we have very precise molecular tools including RT-PCR and sequencing, followed by comparison novel viruses with results on the international database. This work can be done speedily and not only provides information on virus involvement in a specific flock but also enables spread of specific viruses locally or nationally using 'molecular epidemiology'. It should not be forgotten that while these techniques are the ones of choice for the modern 
diagnostician, the availability of live virus through isolation is important for vaccine development or examination of pathogenesis of new types.

\section{Importance of experiments in chickens}

Finally, it is worth emphasising the importance of using chickens (ideally specific pathogen-free) in order to determine the efficacy of vaccines against novel IBV types in addition to investigations into the pathogenesis and pathology relating to such viruses. High disease containment facilities are required to maintain such stock for these essential investigations.

\section{CONCLUSIONS}

IB is highly infectious and maintaining virus-free flocks seems impracticable. The main choice for future strategies in IBV control by vaccination seems to be as follows:

(i) Production of a specific tailored vaccine, produced by molecular technology and designed to protect against the variant challenge virus. This has the advantage that once the virus 'carrier' is established, mechanisms for the insertion of the appropriate S1 spike gene (perhaps with accessory genes for cytokines etc., are now available. However, the question arises as to how many tailored vaccines the bird can take, if the numbers of variants in the challenge become large.

(ii) The use of two different vaccines. This is simple to employ but is not guaranteed to succeed in every case and the mechanisms have not been fully established. However, unless a 'pan-IBV' vaccine is to be produced (which is unlikely), this is a significant and very successful 'stop-gap' approach.

IBV shows viral evolution in action and is likely to remain one step ahead.

\section{REFERENCES}

Asplin FD, Identification of infectious bronchitis in chickens in England. Veterinary Record 1948; 60:485.

Bijlenga G, Cook JKA, Gelb JJr, de Wit JJ. Development and use of the $\mathrm{H}$ strain of avian infectious bronchitis from the Netherlands as a vaccine: a review. Avian Pathology 2004; 33:550-557.

Casais R, Thiel V, Siddell SG, Cavanagh D, Britton P. Reverse genetics system for the avian coronavirus infectious bronchitis virus. Journal of Virology 2001; 75:1235-1236.
Cavanagh D, Casais R, Armesto M, Hodgson T, Izadkhasti S, Davies $M$, Lin F, Tarpey I, Britton P. Manipulation of the infectious bronchitis coronavirus genome for vaccine development and analysis of the accessory proteins. Vaccine $2007 ;$ 26:5558-5562.

Cook JK, Orbell SJ, Woods MA, Huggins MB. Survey of the presence of a new infectious bronchitis virus designated 4/91 (793B). Veterinary Record 1996; 138:178-180.

Cook JKA, Orbell SJ, Woods MA, Huggins MB. Breadth of protection of the respiratory tract provided by two different live attenuated infectious bronchitis vaccines against challenge with infectious bronchitis viruses of heterologous serotypes. Avian Pathology 1999; 28:477-485.

Davelaar FG, Kouwenhoven B, Burger AG. Occurrence and significance of IBV variants strains in egg and broiler production in the Netherlands. Veterinary Quarterly 1984; 6:114-20.

De Wit JJ, Sande H van de. Efficacy of combined vaccines at day of hatching against D388 challenge in SPF and commercial chickens. Proceedings of the 4th International Symposium on Avian Coronaand Pneumoviruses and Complicating Pathogens; 2009; Rauischholzhausen, Giessen. Germany.

Dhinakar Raj G, Jones RC. Protectotypic differentiation of avian infectious bronchitis viruses using an in vitro challenge model. Veterinary Microbiology 1996; 53:239-252.

Dhinakar Raj G, Jones RC. Infectious bronchitis virus: immunopathogenesis of infection in the chicken. Avian Pathology 1997; 26:677-706.

Dolz R, Pujols J, Ordóñez G, Porta R, Majó N. Antigenic and molecular characterization of isolates of the Italy 02 infectious bronchitis virus genotype. Avian Pathology 2006; 35:77-85

Jones RC, Worthington KJ, Capua I, Naylor CJ. Efficacy of live infectious bronchitis vaccines against a novel European genotype Italy 02. Veterinary Record 2005; 156:646-647.

Kusters JG, Niesters HG, Bleumynk-Pluym NM, Davelaar FG, HOrzinek MC, Vander Zeist BA. Molecular epidemiology of infectious bronchitis in the Netherlands. Journal of General Virology 1987; 68:343-52.

Parsons D, Ellis MM, Cavanagh D, Cook JK. Characterisation of an infectious bronchitis virus isolated from vaccinated broiler breeder flocks. Veterinary Record 1992; 131:408-411.

Tarpey I, Orbell SJ, Britton P, Casais R, Hodgson T, Lin F, Hogan E, Cavanagh D. Safety and efficacy of an infectious bronchitis virus used for chicken embryo vaccination. Vaccine 2006; 24:6830-6838.

Worthington $\mathrm{K}$, Currie RE, Jones RC. A reverse transcriptasepolymerase chain reaction survey of infectious bronchitis virus genotypes in Western Europe from 2002 to 2006. Avian Pathology 2008; 37:247-257. 